\title{
Des insulines pour orchestrer la croissance
}

> Pourquoi possédons-nous deux bras de la même longueur? Cette question simple renvoie à l'un des domaines de la biologie du développement qui tente de comprendre comment la croissance de chaque organe est coordonnée pour former un individu adulte bien proportionné. Des travaux effectués chez la drosophile démontrent que Dilp8, une hormone de la famille des insulines/ relaxines, et son récepteur Lgr3 jouent un rôle crucial dans la coordination de la croissance inter-organes et sont requis pour la stabilité développementale. Plus récemment, nous avons démontré que la voie Hippo, par le biais de son effecteur transcriptionnel yki (homologue des facteurs YAP/TAZ chez les mammifères), contrôle les niveaux transcriptionnels de dilp8. Ce contrôle de dilp8 par Yki constitue un pas en avant dans notre compréhension du lien entre la croissance autonome des tissus et la coordination interorganes. <

\section{Notion de croissance}

Les organes en développement coordonnent leur croissance pour former des individus adultes bien proportionnés. Ces mécanismes de coordination sont importants pour maintenir les proportions corporelles en cas de perturbation de la croissance de l'un des organes. Au cours du développement des organismes complexes, la croissance est contrôlée à différents niveaux, de la cellule à l'organisme entier (croissance systémique) en passant par les organes et les tissus. La croissance est définie par une augmentation de la masse d'un tissu ou d'un organisme. Deux paramètres majeurs la caractérisent et déterminent la taille finale d'un adulte. Le premier est la durée de la période de croissance, temps au cours duquel l'organisme grandit. Le deuxième paramètre est le taux (ou vitesse) de croissance de l'orga-

Vignette (Photo (c) Inserm - Loïc Broix).
Emilie Boone, Laura Boulan, Ditte S. Andersen,

Nuria Romero, Pierre Léopold, Julien Colombani

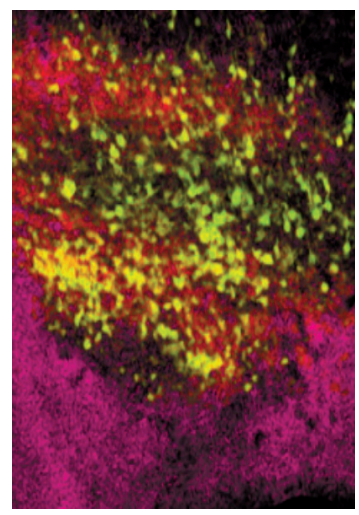

Institut de Biologie de Valrose (iBV), CNRS UMR7277 - Inserm U1091, Université Nice Sophia Antipolis - Parc Valrose - Nice, France.

julien.colombani@unice.fr

nisme. La taille finale d'un organisme est intimement liée à la taille des organes qui le composent. Au cours du développement, chaque organe croît en fonction d'un programme intrinsèque mais s'adapte également aux autres organes/tissus et à l'environnement via des mécanismes extrinsèques.

De par la richesse de ses outils génétiques, la drosophile est un modèle de choix pour l'étude des mécanismes de contrôle de la croissance [1-4] $(\rightarrow)$. Cet insecte présente une période de croissance restreinte aux stades larvaires, tandis que l'adulte ne croît plus. La signalisation insuline/IGF (insulin-like growth factor) contrôle la croissance et le métabolisme des deux types tissulaires qui constituent la larve:

$(\rightarrow)$ Voir les Nouvelles de J. Colombani et al., $m / s n^{\circ} 2$, février 2004, page $141 ; m / s n^{\circ} 3$, mars 2006, page 241 ; $m / s n^{\circ} 11$, novembre 2012, page 918 les tissus mitotiques, ou disques imaginaux ${ }^{1}$, dont les cellules croissent en se divisant, et les tissus endoréplicatifs dont les cellules grossissent sans se diviser. Pendant la métamorphose, la plupart des tissus endoréplicatifs sont histolysés au profit des tissus imaginaux qui se réorganisent pour former les structures de l'adulte. La croissance des tissus imaginaux constitue un excellent modèle pour la croissance tissulaire en général. La plupart des gènes contrôlant la croissance de ces tissus présentent des homologues chez les mammifères, dont beaucoup sont définis comme des oncogènes ou des gènes suppresseurs de tumeur [5]. Un acteur clé de la croissance est la signalisation insuline/IGF, qui est fortement conservée entre vertébrés et insectes. Chez la drosophile, les Dilp 1-7 (drosophila insulin-like peptides) portent à la fois les fonctions métaboliques de l'insuline et les fonctions de croissance des

${ }^{1}$ Chez la drosophile, les disques imaginaux d'aile sont des structures épithéliales spécialisées d'origine ectodermique qui se forment à partir d'un primordium de 10 à 50 cellules chez l'embryon. Les cellules du disque prolifèrent pendant le développement larvaire pour atteindre un nombre de 30000 à 50000 cellules à la transition larvaire-pupale. Lors de la métamorphose, les disques sont remodelés pour former les appendices de l'adulte. 


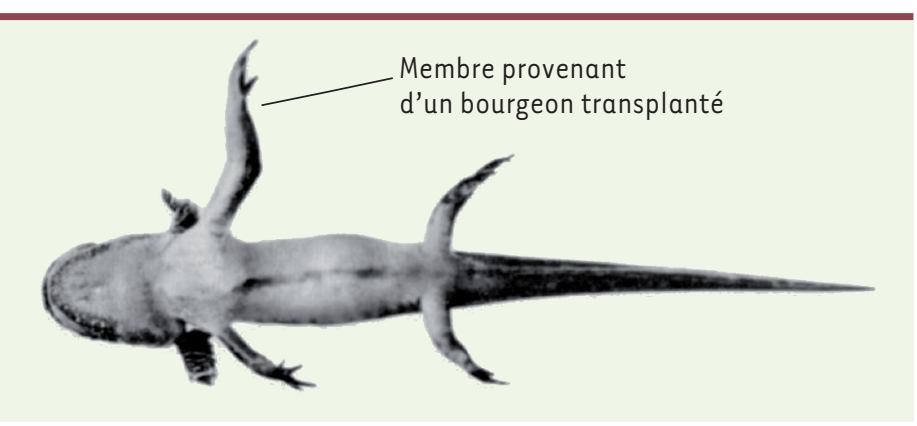

Figure 1. La taille des membres est génétiquement programmée chez la salamandre. Un bourgeon de membre d'une grande espèce de salamandre (Ambystoma trigrinum) est transplanté sur un embryon d'une plus petite espèce (Ambystoma punctatum). Le membre transplanté grandit plus que les membres de l'hôte, atteignant la taille qu'il aurait dû atteindre chez Ambystoma trigrinum (๔ photographie de R.G. Harrison, 1969).

IGF vertébrés. L'étude des nombreux mutants de la voie du récepteur de l'insuline chez la drosophile a clairement établi l'importance de ce mécanisme hormonal dans le contrôle de la croissance et du métabolisme en fonction des conditions environnementales [6].

La notion de contrôle de la taille des organes par des mécanismes intrinsèques provient d'expériences de transplantation de bourgeons de membres entre des espèces de salamandres du genre Ambystoma de différentes tailles [7, 8]. Dans les années 1920, Harrison et al. avaient déjà montré que les bourgeons de membres provenant de deux espèces de taille différente et transplantés dans des receveurs se développent en fonction de la taille d'origine du membre des donneurs et non de celle des receveurs (Figure 1). Cette expérience indique que, quel que soit l'environnement (facteurs circulants, tissus voisins) dans l'organisme hôte, le programme de croissance intrinsèque au tissu greffé est dominant. Ce type de contrôle autonome est aussi observé chez la drosophile. En 1985, Bryant et Lenvinson ont montré que des disques imaginaux de larve encore immatures peuvent se développer dans l'abdomen de femelles adultes (un environnement permissif pour la croissance) et atteignent une taille similaire à celle observée à la fin du développement larvaire. Cette expérience confirme l'existence d'un mécanisme de détermination intrinsèque de la taille des disques chez la drosophile $[9,10]$.

Lorsque la croissance d'un organe est affectée par manipulation génétique ou chirurgicale, ou par des variations développementales [11], une croissance régénérative se met en place compensant la perte de masse. L'organe lésé induit également un retard de développement lui permettant d'atteindre sa taille finale et affecte la croissance des organes intacts, qui adaptent leur croissance à celle de l'organe lésé. Le cas de la croissance des organes pairs est très intriguant. Quels processus entrent en jeu pour permettre à deux organes pairs, en l'absence de tout contact physique, de posséder à la fin du développement une taille remarquablement similaire? Comment, lors d'une lésion sur l'un des organes pairs, l'organe contralatéral non lésé perçoit-il la lésion et s'adapte-t-il en ralentissant son développement? Enfin, il faut noter que les différents organes qui constituent le corps suivent des courbes de croissance différentes, en fonction des principes d'allométrie qui permettent le respect des proportions pour une espéce donnée [12]. La coordination de ces differents rythmes de croissance fait donc appel à des contrôles à la fois spatiaux et temporels qui sont encore largement inconnus.

Ces aspects physiologiques de la croissance posent trois questions fondamentales. Comment un organe mesure-t-il sa propre taille? Comment la croissance est-elle coordonnée entre les organes? Quels sont les mécanismes qui coordonnent la croissance des organes et l'horloge développementale?

\section{Coordination de la croissance avec le programme développemental}

Chez la drosophile, une altération de la croissance du disque imaginal d'aile par chirurgie ou par modifications génétiques entraîne un retard développemental [13-17]. Ce retard de développement a servi de point de départ pour un crible génétique qui a permis l'identification du gène codant pour Dilp8, une hormone peptidique de la famille des insulines/relaxines, sécrétée par les tissus dont la croissance est altérée [18]. Dilp8 agit au niveau du système nerveux central où sont produites les hormones contrôlant l'horloge développementale. Lorsqu'il est induit, Dilp8 bloque la synthèse de l'ecdysone (l'hormone stéroïde de mue), empêchant ainsi les larves de progresser vers le stade pupal $\left.\right|^{2}$. De cette façon, Dilp8 rallonge la période larvaire de croissance et permet au tissu lésé d'effectuer une croissance réparatrice [18, 19]. La récente identification de $\mathrm{Lgr} 3$ (leucine-rich repeat $G$ protein-coupled receptor 3 ) comme récepteur de Dilp8 a permis de mieux comprendre son action. Lgr3 est un récepteur couplé aux protéines $G(G P C R)$, analogue à la sous-famille des récepteurs des relaxines chez les vertébrés. L'inactivation de Lgr3 par ARN interférent (ARNi) permet de remédier au retard de développement induit par la surexpression de Dilp8 [20]. Lgr3 est présent dans une sous-population de neurones dont deux seulement sont requis pour l'induction du retard développemental par Dilp8 (appelés growth coordinating Lgr3-positive neurons ou neurones $\mathrm{GCL}$ ). Les neurones GCL semblent contrôler les niveaux d'ecdysone par une interaction physique avec des neurones intermédiaires à PTTH (prothoracicotropic hormone) ${ }^{3}$ qui projettent leurs axones sur la glande en anneau, le site de production de l'ecdysone [20-24].

${ }^{2}$ Le cycle de vie de la drosophile se divise en quatre phases durant lesquelles les individus prennent des morphologies très différentes : l'œuf (stade embryonnaire), la larve (stade larvaire), la pupe (stade pupal) et l'imago (stade adulte).

${ }^{3} \mathrm{PTH}$ est une hormone qui intervient dans la régulation et la production de l'ecdysone. 
Dilp8 représente donc un cas particulier au sein de la famille des drosophila insulin-like peptides. Contrairement aux autres Dilp (Dilpl-7), qui promeuvent la croissance en induisant une signalisation via le récepteur de l'insuline InR, Dilp8 inhibe la croissance via son récepteur Lgr3. Cette signalisation particulière impliquant Lgr3 explique le rôle physiologique très différent de Dilp8 et notamment son implication dans la coordination de la croissance entres organes [6].

\section{Coordination de la croissance entre organes et robustesse développementale}

Le maintien de la taille des différents organes en proportion avec la taille finale de l'organisme est un processus développemental complexe encore mal connu. Chez la drosophile, la réduction artificielle du taux de croissance du disque imaginal d'aile ralentit la croissance des autres disques [25]. Un apport d'ecdysone dans la nourriture des larves bloque l'ajustement des taux de croissance entre disques, suggérant qu'un réglage fin des taux circulants d'ecdysone est nécessaire à cette coordination inter-organes [25-27]. II semble que Dilp8 participe également à cette coordination en activant la voie de la nitric oxyde synthase (NOS) par le récepteur Lgr3 dans la glande en anneau [28]. Si la baisse des niveaux systémiques d'ecdysone contribue à ralentir la croissance des tissus non lésés, il reste cependant à comprendre par quel mécanisme les tissus lésés eux-même échappent à cette inhibition de la croissance.

La coordination de croissance entre les organes est particulièrement précise dans le cas des organes pairs présentant une symétrie bilatérale [29] $(\rightarrow)$. Chez la drosophile, la symétrie bilatérale des ailes est essentielle pour une bonne aptitude au vol. Des événements de déviation par rapport à la symé- $(\rightarrow)$ Voir le Forum de V. Debat, $m / s n^{\circ} 8-9$, août-septembre 2016, page 774 trie parfaite sont observés au cours du développement des organismes et correspondent à un bruit développemental appelé asymétrie fluctuante $^{4}[29,30]$. L'étude comparative des ailes droite et gauche chez la drosophile adulte permet l'observation de cette asymétrie fluctuante et constitue le reflet d'une stabilité développementale plus ou moins altérée [30]. Une formule fondée sur la variance de la différence de surface des ailes droite et gauche de chaque individu permet de calculer un index d'asymétrie fluctuante qui quantifie cette variabilité développementale. Dilp8 est un acteur important de ce processus puisque les animaux mutants pour dilp8 présentent une asymétrie des ailes importante (Figure 2). En lien avec cette idée, l'inactivation de lgr3 dans les neurones GCL induit également une asymétrie fluctuante $[19,20]$. Dilp8 semble donc agir directement sur le système nerveux central via Lgr3 pour assurer la stabilité développementale.

Si des mécanismes systémiques semblent assurer la coordination de la croissance par l'intermédiaire de Dilp8 et de son récepteur au niveau du système nerveux central, on ne peut exclure pour l'instant que Dilp8 agisse également de façon autonome sur la croissance du

${ }^{4}$ L'asymétrie fluctuante, qui correspond aux différences entre les côtés droit et gauche d'un organisme à symétrie bilatérale, reflète la limite de précision du développement.

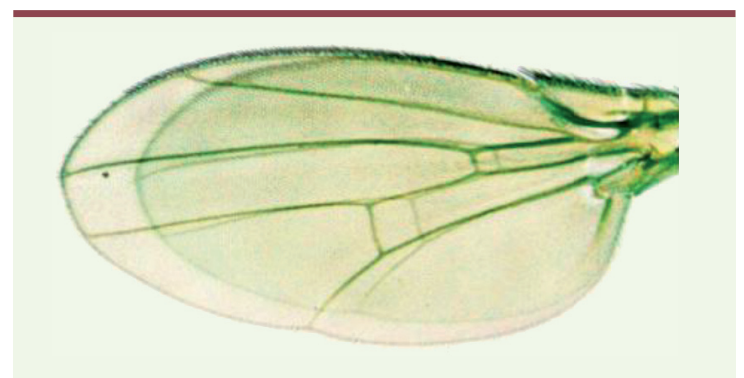

Figure 2. Défaut de symétrie bilatérale chez le mutant dilp8. L'aile de drosophile, un tissu facilement accessible, est utilisée pour suivre la coordination de la croissance entre les organes. Cette image des ailes superposées d'une même mouche, mutante pour le gène dilp8, illustre la perte de symétrie bilatérale entre les organes gauche et droit d'un même individu (c) Emilie Boone).

disque. L'expression de lgr3 n'étant pas détectable au sein des disques imaginaux $[20,21]$, Dilp8 pourrait agir de façon autonome via un autre récepteur. Un test de capture ligand-récepteur suivi d'une analyse par spectrométrie de masse a montré que Dilp8 peut se lier in vitro au récepteur unique de l'insuline $(\ln R)$ [21]. Dilp8 pourrait donc contrôler la croissance en se fixant à I'InR et en modulant par exemple la capacité des autres Dilp à activer la croissance des tissus imaginaux via ce récepteur.

\section{Croissance tissulaire et production de Dilp8}

La coordination inter-organes sous-entend que chaque tissu puisse évaluer sa propre croissance/taille et qu'il communique cette information à son environnement. Les mutants dilp 8 présentent une augmentation du bruit développemental qui se traduit par une altération de la symétrie bilatérale des organes. Mais quel est le lien entre la croissance des tissus et les niveaux de Dilp8 qu'ils sécrètent?

Nous avions précedemment établi le rôle de la voie de signalisation de la Jun $N$-terminal kinase (JNK) dans l'induction de dilp8 en réponse à une modification importante/pathologique de la croissance tissulaire [18]. Cependant, la voie JNK n'est pas induite en condition normale de croissance, suggérant un autre mode de contrôle plus physiologique pour dilp8 au cours du développement. Nous avons plus récemment établi que, parallèlement au contrôle exercé par la voie JNK, la voie de signalisation Hippo/Yki ${ }^{5}$ contrôle les

${ }^{5}$ Hippo (Hpo) est un suppresseur de tumeur (Mstl/2Mst2 chez les mammifères). $y_{\mathrm{ki}}$ (pour Yorkie) est une oncoprotéine (YAP [Yes-associated protein] et TAZ [transcriptional coactivator with PDZ-binding motif] chez les mammifères). 


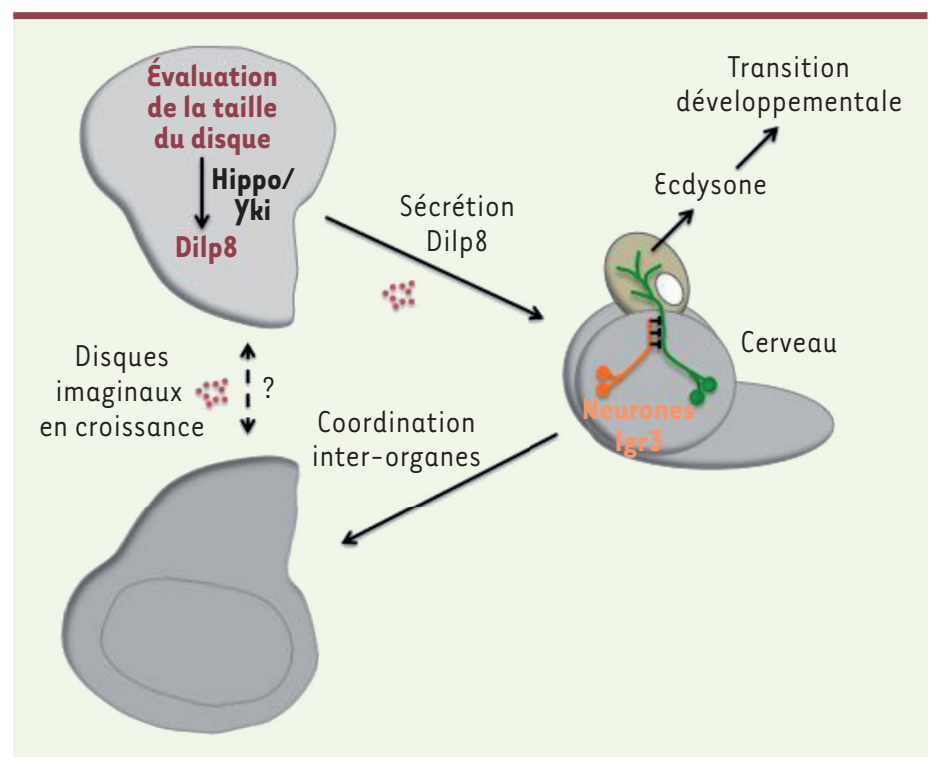

Figure 3. Dilp8 est impliqué dans la coordination inter-organes et l'horloge développementale. La régulation de l'expression de dilp8 par la voie de signalisation Hippo/Yki est requise pour le contrôle de la stabilité développementale et la détermination fine de la taille des organes.

niveaux transcriptionnels de dilp8 en condition normale de croissance des tissus [31]. La voie Hippo/Yki est conservée au cours de l'évolution et joue un rôle clé dans le contrôle de la taille des organes en couplant les paramètres biomécaniques des tissus (densité cellulaire et contraintes physiques) avec la croissance et la prolifération cellulaire [32,33] $(\rightarrow)$. Le dérèglement de la voie Hippo est impliqué dans de nombreux cancers humains [5]. Les co-activateurs transcriptionnels de la voie, Yorkie ( $\mathrm{Yki}$ ) $(\rightarrow)$ Voir la Synthèse de P.L. Bardet, $m / s$ $n^{\circ} 3$, mars 2009, page 253 et Scalloped $(\mathrm{Sd})^{6}$, contrôlent directement l'expression de dilp8 en se liant à un élément de réponse à Hippo (HRE) présent dans le promoteur de dilp8 [31]. La validation fonctionnelle de cet élément a été réalisée d'une part in vitro, en démontrant une interaction physique directe, puis in vivo en suivant l'activation transcriptionnelle de dilp8 par Yki/ $\mathrm{Sd}$. Dans le but de valider fonctionnellement le rôle de la voie Hippo dans le contrôle de dilp8, un mutant dilp8 a été généré par CRISPR/Cas9 $[34,35](\rightarrow)$. Les sites de fixation pour $\mathrm{Sd} / \mathrm{Yki}$ dans le HRE endogène du promoteur dilp8 ont été mutés, créant ainsi un allèle du gène dilp8 insensible au contrôle trans- $(\rightarrow)$ Voir la Synthèse $\mathrm{m} / \mathrm{s} \mathrm{n}^{\circ} 12$, décembre 2014, page 1066 de H. Gilgenkrantz, criptionnel par Hippo/Yki. La modification du HRE au locus dilp8 induit une asymétrie fluctuante chez les adultes similaire à celle observée pour le mutant dilp8 nul (dépourvu du gène). Ceci démontre un couplage entre la voie de signalisation Hippo/Yki, acteur clé de la croissance autonome des tissus, et l'expression de dilp8 in vivo, et établit l'importance de ce contrôle pour assurer la stabilité développementale et la coordination inter-organes (Figure 3) [31].

\footnotetext{
${ }^{6}$ Homologue de TEF-1 (transcriptional enhancer factor 1 ) chez l'homme.
}

\section{Conclusion}

L'axe Dilp8/Lgr3 joue donc un rôle central pour assurer un développement harmonieux chez la drosophile. Dilp8 et son récepteur Lgr3 régulent au moins deux mécanismes importants pour l'émergence d'adultes bien proportionnés. D’une part, la voie JNK régule la transcription de dilp8 en réponse à différentes conditions de stress tissulaire, représentant un point de contrôle important pour la régénération tissulaire. Ceci se traduit par une forte induction transcriptionelle lors d'une croissance ralentie, d'une croissance néoplasique ou durant la régénération tissulaire, qui provoque un retard de développement et un allongement de la période de croissance larvaire. Le promoteur de dilp8 contient des sites de liaison prédits pour le facteur de transcription de la voie JNK, API (activator protein 1), qui pourraient permettre une régulation directe de l'expression de dilp8 par cette voie. Néanmoins, les mutants viables pour la voie JNK ne présentent pas d'asymétrie fluctuante, indiquant que cette voie ne contrôle pas la coordination de la croissance en condition normale de développement. Par ailleurs, Dilp8 est requis pour la coordination fine de la croissance inter-organes en conditions physiologiques de développement, puisqu'un mutant nul pour dilp8 présente une augmentation de l'asymétrie fluctuante. Ceci suggère donc que l'état de maturation/croissance des tissus influe directement sur les niveaux d'expression de dilp8. Le lien entre croissance autonome et niveaux de Dilp8 est établi par la régulation directe du promoteur de dilp8 par la voie Hippo/ yki, assurant ainsi la coordination de la croissance des organes et la stabilité développementale. Par ailleurs, les tissus imaginaux en croissance subissent des déformations et des contraintes physiques au cours du développement. La voie de signalisation Hippo intègre de nombreuses informations telles que la polarité cellulaire, l'adhérence cellulaire et les forces mécaniques, qu'elle traduit sous forme de croissance. Le couplage entre l'expression de dilp8 et la voie de signalisation Hippo/yki ouvre donc la possibilité d'une coordination de la croissance en réponse aux forces mécaniques.

Il est intéressant d'évoquer enfin que le circuit neuronal formé par les neurones GCL et contrôlant la production d'ecdysone chez la drosophile possède certaines caractéristiques de l'axe hypothalamushypophyse chez l'homme. L'hypothalamus intègre et transmet des informations sur l'état de croissance des organes à l'hypophyse qui produit des messagers hormonaux (dont l'hormone de croissance) [36-38]. Des 
récepteurs de type relaxine sont exprimés au sein de l'hypothalamus, suggérant que des relaxines pourraient être sécrétées par les tissus en croissance et informer l'hypothalamus sur leur état de croissance. Dès lors, un axe homologue de l'axe Dilp8/Lgr3 pourrait représenter un mécanisme de contrôle de la stabilité développementale chez les vertébrés. $\diamond$

\section{SUMMARY}

\section{Some insulins to orchestrate growth}

Body size is an intrinsic property of living organisms that is intimately linked to the developmental program to produce fit individuals with proper proportions. Final size is the result of both genetic determinants and sophisticated mechanisms adapting size to available resources. Even though organs grow according to autonomous programs, some coordination mechanisms ensure that the different body parts adjust their growth with the rest of the body. In Drosophila, Dilp8, a hormone of the Insulin/Relaxin family is a key player in this inter-organs coordination and is required together with its receptor Lgr3 to limit developmental variability. Recently, the transcriptional co-activator yki (homologue of YAP/TAZ factors in mammals) was shown to regulate dilp8 expression and contribute to the coordination of organ growth in Drosophila. $\diamond$

\section{LIENS D'INTÉRÊT}

Les auteurs déclarent n'avoir aucun lien d'intérêt concernant les données publiées dans cet article.

\section{RÉFÉRENCES}

1. Colombani J, Arquier N, Leopold P. Les mouches gardent la ligne : slimfast, le corps gras et le contrôle humoral de la croissance. Med Sci (Paris) 2004 ; $20: 141$-3.

2. Colombani J, Bianchini L, Layalle $S$, et al. Stéroïdes, insuline et croissance : les mouches dopent la recherche. Med Sci (Paris) 2006 ; 22 : 241-3.

3. Colombani J, Andersen DS, Leopold P. Atteindre la bonne taille... Med Sci (Paris) 2012 ; 28 : 918-9.

4. Andersen DS, Colombani J, Leopold P. Coordination of organ growth: principles and outstanding questions from the world of insects. Trends Cell Biol $2013 ; 23: 336-44$.

5. Hariharan IK, Bilder D. Regulation of imaginal disc growth by tumor-suppressor genes in Drosophila. Annu Rev Genet 2006 ; 40 : 335-61.

6. Nassel DR, Vanden Broeck J. Insulin/IGF signaling in Drosophila and other insects: factors that regulate production, release and post-release action of the insulin-like peptides. Cell Mol Life Sci $2016 ; 73: 271-90$.

7. Harrison RG. Some unexpected results of the heteroplastic transplantation of limbs. Proc Natl Acad Sci USA $1924 ; 10: 69-74$.

8. Twitty VC, Schwind JL. The growth of eyes and limbs transplanted heteroplastically between two specis of Amblistoma. J Exp Zool 1931; 59 : 61-86.

9. Bryant PJ, Simpson P. Intrinsic and extrinsic control of growth in developing organs. $\varrho$ Rev Biol $1984 ; 59: 387-415$.

10. Bryant PJ, Levinson P. Intrinsic growth control in the imaginal primordia of Drosophila, and the autonomous action of a lethal mutation causing overgrowth. Dev Biol $1985 ; 107$ : 355-63.

11. Hariharan IK, Serras F. Imaginal disc regeneration takes flight. Curr Opin Cell Biol 2017 ; 48 : $10-6$.

12. Stern DL, Emlen DJ. The developmental basis for allometry in insects. Development $1999 ; 126$ : 1091-101.

13. Simpson P, Berreur P, Berreur-Bonnenfant J. The initiation of pupariation in Drosophila: dependence on growth of the imaginal discs. J Embryol Exp Morphol $1980 ; 57$ : 155-65.

14. Poodry CA, Woods DF. Control of the developmental timer for Drosophila pupariation. Roux's Archives of Developmental Biology 1990 ; 199 : 219-27.

15. Stieper BC, Kupershtok M, Driscoll MV, et al. Imaginal discs regulate developmental timing in Drosophila melanogaster. Dev Biol 2008 ; 321 : 18-26.

16. Smith-Bolton RK, Worley MI, Kanda H, et al. Regenerative growth in Drosophila imaginal discs is regulated by Wingless and Myc. Dev Cell $2009 ; 16: 797-809$.
17. Hariharan IK. How growth abnormalities delay "puberty" in Drosophila. Sci Signal $2012 ; 5$ : pe27.

18. Colombani J, Andersen DS, Leopold P. Secreted peptide Dilp8 coordinates Drosophila tissue growth with developmental timing. Science 2012 ; 336 : 582-5.

19. Garelli A, Gontijo AM, Miguela V et al. Imaginal discs secrete insulin-like peptide 8 to mediate plasticity of growth and maturation. Science 2012 ; $336: 579-82$.

20. Colombani J, Andersen DS, Boulan L, et al. Drosophila Lgr3 couples organ growth with maturation and ensures developmental stability. Curr Biol $2015 ; 25: 2723-9$.

21. Garelli A, Heredia F, Casimiro AP, et al. Dilp8 requires the neuronal relaxin receptor Lgr3 to couple growth to developmental timing. Nat Commun 2015 ; $6: 8732$.

22. Vallejo DM, Juarez-Correno S, Bolivar J, et al. A brain circuit that synchronizes growth and maturation revealed through Dilp8 binding to Lgr3. Science 2015 ; 350 : aac6767.

23. Jaszczak JS, Wolpe JB, Bhandari R, et al. Growth coordination during Drosophila melanogaster imaginal disc regeneration is mediated by signaling through the relaxin receptor Lgr3 in the prothoracic gland. Genetics 2016 ; $204: 703-9$.

24. Jaszczak JS, Halme A. Arrested development: coordinating regeneration with development and growth in Drosophila melanogaster. Curr Opin Genet Dev $2016 ; 40: 87-94$.

25. Parker NF, Shingleton AW. The coordination of growth among Drosophila organs in response to localized growth-perturbation. Dev Biol $2011 ; 357$ : 318-25.

26. Herboso L, Oliveira MM, Talamillo A, et al. Ecdysone promotes growth of imaginal discs through the regulation of Thor in D. melanogaster. Sci Rep $2015 ; 5: 12383$.

27. Gokhale RH, Hayashi T, Mirque CD, et al. Intra-organ growth coordination in Drosophila is mediated by systemic ecdysone signaling. Dev Biol $2016 ; 418$ : $135-45$.

28. Jaszczak JS, Wolpe JB, Dao AQ, et al. Nitric oxide synthase regulates growth coordination during Drosophila melanogaster imaginal disc regeneration. Genetics $2015 ; 200: 1219-28$.

29. Debat V. Symmetry is beauty - or is it ? Grandeur et décadence de l'asymétrie fluctuante. Med Sci (Paris) 2016 ; $32: 774-80$.

30. Debat V, Peronnet F. Asymmetric flies: The control of developmental noise in Drosophila. Fly $2013 ; 7: 70-7$.

31. Boone $\varepsilon$, Colombani J, Andersen DS, et al. The Hippo signalling pathway coordinates organ growth and limits developmental variability by controlling dilp8 expression. Nat Commun 2016; $7: 13505$.

32. Bardet PL. La voie Hippo contrôle la croissance des organes au cours du développement. Med Sci (Paris) $2009 ; 25: 253-7$.

33. Yu FX, Zhao B, Guan KL. Hippo pathway in organ size control, tissue homeostasis, and cancer. Cell $2015 ; 163: 811-28$.

34. Gilgenkrantz H. La révolution des CRISPR est en marche. Med Sci (Paris) $2014 ; 30: 1066-9$.

35. Baena-Lopez LA, Alexandre C, Mitchell A, et al. Accelerated homologous recombination and subsequent genome modification in Drosophila. Development 2013 ; 140 : 4818-25.

36. Roselló-Díez A, Joyner AL. Regulation of long bone growth in vertebrates; It is time to catch up. Endocr Rev $2015 ; 36: 646-80$.

37. McGowan BM, Stanley SA, Donovan J, et al. Relaxin-3 stimulates the hypothalamic-pituitary-gonadal axis. Am J Physiol Endocrinol Metab 2008 ; 295 : ع278-86.

38. McGowan BM, Minnion JS, Murphy KG, et al. Relaxin-3 stimulates the neuroendocrine stress axis via corticotrophin-releasing hormone. J Endocrinol $2014 ; 221: 337-46$. 